\title{
Collisional process on Comet 9/P Tempel 1: mass loss of its dust and ice by impacts of asteroidal objects and its collisional history
}

\author{
Satoru Yamamoto ${ }^{1}$, Koji Wada $^{2}$, Hiroshi Kobayashi ${ }^{2}$, Hiroshi Kimura ${ }^{2}$, Masateru Ishiguro ${ }^{3}$, and Takafumi Matsui ${ }^{1}$ \\ ${ }^{1}$ Graduate School of Frontier Science, Univ. of Tokyo, Japan \\ ${ }^{2}$ Institute of Low Temperature Science, Hokkaido Univ., Japan \\ ${ }^{3}$ National Astronomical Observatory of Japan
}

(Received September 4, 2008; Revised November 7, 2008; Accepted November 11, 2008; Online published February 12, 2010)

\begin{abstract}
We have studied the collisional process on the nucleus of Comet 9/P Tempel 1 (T1) and estimated the mass loss of surface materials due to the impacts of asteroidal objects. The mass loss rate is around $\sim 2 \times 10^{5} \mathrm{~kg} \mathrm{yr}^{-1}$, which is smaller than that of water sublimation of $\sim 6 \times 10^{9} \mathrm{~kg} \mathrm{yr}^{-1}$. We then estimated the number density of craters formed by asteroid impacts to infer the collisional history of $\mathrm{T} 1$. We found that the time necessary to accumulate the crater population on $\mathrm{T} 1$ is as long as $\sim$ a few $10^{4}$ years, suggesting that $\mathrm{T} 1$ crossed the asteroid belt region more than $\sim$ a few $10^{4}$ years ago, though this estimated time may be reduced to several thousands of years by taking into account the influence of the sublimation process on the crater size. Alternatively, the total period of the recent orbit, in which the perihelion distance is small enough to cause significant sublimation by erasing a large number of small craters, may be less than $\sim 2 \times 10^{3}$ years.
\end{abstract}

Key words: Comets, impact cratering, asteroid, dust mantle.

\section{Introduction}

Short-period comets were originally trans-Neptunian objects formed from dust and ice in the outer cold region of the primordial solar nebula. When such a comet leaves the trans-Neptunian region and approaches the Sun, volatile components of its nucleus begin to sublime and form a dust mantle on the surface. Vapor pressure of the sublimating components preferentially carries the dust having a high surface-to-mass ratio off the surface. The sublimation process also leads to chemical differentiation of a nucleus owing to preferential sublimation losses of highly volatile materials in the deeper parts of the nucleus. In addition, trapped primordial volatile gasses are released when pristine amorphous water ices just below the dust mantle crystallize to form a crystalline ice crust. Heating caused by radioactive decay might have altered even the central region of the nucleus. Therefore, primordial dust and ice would remain only local regions well below the surface and well above the center of the nucleus (e.g., Jewitt, 1992; Meech, 2000; Prialnik et al., 2004, 2008). This scenario, which is hereafter called the standard model, has turned out to describe well the nucleus of Comet 9P/Tempel 1 (T1), the target of NASA's Deep Impact (DI) mission: (1) a low thermal inertia of its surface estimated from thermal maps of the nucleus indicates the presence of a dust mantle (A'Hearn et al., 2005); (2) the existence of large grains in a dust trail along the trajectory of the comet would result from the preferential elimination of small-sized dust

Copyright (C) The Society of Geomagnetism and Earth, Planetary and Space Sciences (SGEPSS); The Seismological Society of Japan; The Volcanological Society of Japan; The Geodetic Society of Japan; The Japanese Society for Planetary Sciences; TERRAPUB.

doi:10.5047/eps.2008.11.003 during the formation of a dust mantle (Sykes and Walker, 1992; Reach et al., 2007); (3) the optical and infrared properties of dust excavated by the DI event are explained well by the existence of a dust mantle composed of compact aggregates, below which fluffy aggregates are also embedded with icy volatiles (Yamamoto et al., 2008); (4) near-infrared spectroscopic observations of volatiles excavated by the DI event revealed thermal processing of icy layers below a dust mantle (Mumma et al., 2005); (5) the sublimation rate of $6 \times 10^{27}$ water molecules s ${ }^{-1}\left(\sim 180 \mathrm{~kg} \mathrm{~s}^{-1}\right)$ from the T1 nucleus before the DI event implies that the top surface of several meters could have been lost simply by the sublimation process (e.g., Schleicher et al., 2006). In addition, the standard model has been supported by numerical studies on thermal evolution of the T1 nucleus: the formation of a dust mantle with chemical differentiations of the T1 nucleus (De Sanctis et al., 2007); a crystalline ice crust with a thickness of 40-240 m below a dust mantle (Bar-Nun et al., 1989).

In the above scenario, sublimation is an important process by which a comet nucleus can evolve. In addition to the sublimation process, however, impacts by asteroidal bodies may play a vital role in the evolution of the nucleus (e.g., Fernández, 1981). This is the most likely case for T1 because the present orbit of $\mathrm{T} 1$ crosses the asteroid belt region. Indeed, many crater-like features have been found on the T1 surface (Thomas et al., 2007). Thus, a study on the degree of the impact process is the key to understanding the evolution of the T1 nucleus. Furthermore, an application of crater chronology to the $\mathrm{T} 1$ nucleus may allow us to infer the collisional history on $\mathrm{T} 1$ and consequently provide us with clues about the orbital evolution of T1 in the past.

There are several studies on the impacts of asteroids onto the $\mathrm{T} 1$ nucleus, the results of which strongly depend on the 
Table 1. Parameters used in this study.

\begin{tabular}{lccl}
\hline & $9 \mathrm{P} / \mathrm{Tempel} 1$ & Impactor & Reference \\
\hline$R_{\mathrm{T}}$ & $3 \mathrm{~km}$ & A'Hearn et al. (2005) \\
$A$ & $1.1 \times 10^{8} \mathrm{~m}^{2}$ & $4 \pi R_{\mathrm{T}}^{2}$ \\
$\rho_{\mathrm{t}}$ & $450 \mathrm{~kg} \mathrm{~m}^{-3}$ & & Davidsson et al. $(2007)$ \\
$g$ & $5 \times 10^{-4} \mathrm{~m} \mathrm{~s}^{-2}$ & & A'Hearn et al. $(2005)$ \\
$v_{\mathrm{esc}}$ & $1.5 \mathrm{~m} \mathrm{~s}^{-1}$ & & $\sqrt{8 \pi \rho_{\mathrm{t}} G / 3} R_{\mathrm{T}}$ \\
$P_{\mathrm{i}}^{\dagger}$ & $2 \times 10^{-24} \mathrm{~m}^{-2} \mathrm{yr}^{-1}$ & & Gil-Hutton (2000) \\
$\rho_{\mathrm{i}}$ & & $3500 \mathrm{~kg} \mathrm{~m}^{-3}$ & Hawkes and Eaton (2004) \\
$v_{\mathrm{i}}^{\dagger}$ & & $10 \mathrm{~km} \mathrm{~s}^{-1}$ & Gil-Hutton (2000) \\
\hline
\end{tabular}

$R_{\mathrm{T}}$ : radius of nucleus, $A$ : surface area of nucleus, $\rho_{\mathrm{t}}$ : density of nucleus, $g$ : surface gravity of nucleus, $v_{\mathrm{esc}}$ : escape velocity, $P_{\mathrm{i}}$ : intrinsic collision probability, $\rho_{\mathrm{i}}$ : density of impactor, $v_{\mathrm{i}}$ : impact velocity. ${ }^{\dagger}$ This value is derived for the current orbit of T1 with semimajor axis of 3.1 AU, eccentricity of 0.52 , and inclination of $10.5^{\circ}$ (Gil-Hutton, 2000).

model of the asteroidal population (e.g., Hawkes and Eaton, 2004; Gronkowski, 2007; Yamamoto et al., 2008). For example, Hawkes and Eaton (2004) estimated the mass flux of asteroids into the $\mathrm{T} 1$ nucleus to range from $\sim 10^{2}$ to $10^{7} \mathrm{~kg}$ $\mathrm{yr}^{-1}$, depending on the model they used. While the previous models are based on the data of asteroids with sizes larger than $\sim 100 \mathrm{~m}$, small asteroids with sizes of less than $100 \mathrm{~m}$ play an important role in the collisional history of the $\mathrm{T} 1$ nucleus. The asteroidal model by O'Brien and Greenberg (2005) or O'Brien et al. (2006) (hereafter OGR model) is a self-consistent numerical model for the main-belt and nearEarth Asteroid (NEA) populations of small asteroids. These researchers developed the model based on a wide range of observational constraints, including small crater population on NEAs (see OGR model). Michel et al. (2009) reported that the asteroid population model by Bottke et al. (2005) (hereafter BTK model) may actually be more realistic than the OGR model to explain the crater population on NEA (25143) Itokawa. We are, therefore, convinced that either the OGR model or the BTK model is the best available model for the population of small asteroids colliding with comet nuclei. On the basis of these models, we have studied collisional and sublimation processes on the T1 nucleus to assess the mass loss rate of surface materials by impacts of asteroids and to infer the collisional history of the T1 nucleus.

\section{Mass Loss of Surface Materials from T1 due to Impacts of Asteroidal Objects}

We first estimate the mass loss by impacts of asteroids by assuming that both $\mathrm{T} 1$ and asteroids are homogeneous spheres. The rate of the excavated mass $M_{\mathrm{col}}$ by impacts of asteroids into a comet with radius $R_{\mathrm{T}}$ is given as:

$$
M_{\mathrm{col}}=\int_{r_{\min }}^{r_{\max }} P_{\mathrm{i}}\left(R_{\mathrm{T}}+r\right)^{2} n(r) m_{\mathrm{e}}(r) d r,
$$

where $P_{\mathrm{i}}$ is the intrinsic collision probability of T1, $n(r) d r$ is the number of asteroids with radii ranging from $r$ to $r+d r, r_{\min }$ and $r_{\max }$ are the minimum and maximum radii of asteroids impacting into T1, respectively, and $m_{\mathrm{e}}(r)$ is the total mass of impact ejecta escaping from the T1 surface by an impact of an asteroid with radius $r$.

The description of $n(r)$ in the OGR model was not given explicitly in O'Brien and Greenberg (2005) nor O'Brien et al. (2006). Thus, we determine $n(r)$ for the OGR model using the cumulative number $N_{\text {as }}(d)$ of asteroids with larger than a given diameter $d$, as shown in figure 3 of Michel et al. (2009). From this figure we fit $N_{\text {as }}(d)$ with an assumption of a power-law size distribution as:

$$
N_{\text {as }}(d)=3.3 \times 10^{13}\left(\frac{d}{1 \mathrm{~m}}\right)^{-2.88}, \quad \text { for } d \leq 100 \mathrm{~m} \text {. }
$$

Considering $d=2 r$, we obtain

$$
n(r)=-\frac{d N_{\mathrm{as}}(d)}{d r}=1.3 \times 10^{13}\left(\frac{r}{1 \mathrm{~m}}\right)^{-3.88} \mathrm{~m}^{-1} .
$$

The $m_{\mathrm{e}}(r)$ can be obtained from Eq. (A.7) in Appendix as:

$$
m_{\mathrm{e}}(r)=1.0 \times 10^{8}\left(\frac{r}{1 \mathrm{~m}}\right)^{3} \quad \mathrm{~kg},
$$

where we assume the impact velocity $v_{\mathrm{i}}$, the escape velocity $v_{\text {esc }}$, the density of impactor $\rho_{\mathrm{i}}$, and the density of comet nucleus $\rho_{\mathrm{t}}$ as listed in Table 1 , respectively (we refer to Table 1 for the values of parameters that will appear in the text hereafter).

Substituting Eqs. (3) and (4) into Eq. (1), we obtain

$$
M_{\mathrm{col}} \sim 1.9 \times 10^{5}\left(\frac{r_{\max }}{1 \mathrm{~m}}\right)^{0.12} \mathrm{~kg} \mathrm{yr}^{-1},
$$

where we assume $r_{\min } \ll r_{\max } \ll R_{\mathrm{T}}$. Equation (5) indicates that the mass loss rate by impacts of asteroidal objects is around $\sim 2 \times 10^{5} \mathrm{~kg} \mathrm{yr}^{-1}$ for asteroids of $r_{\max }=1 \mathrm{~m}$, which would produce the largest crater with a radius of $R_{\text {cr }} \sim 200$ m observed on T1 (cf. Eq. (11)). Alternatively, the mass loss rate of water sublimation near the perihelion is $\sim 6 \times 10^{9} \mathrm{~kg} \mathrm{yr}^{-1}$, which is derived from the observational value of $6 \times 10^{27}$ molecules s ${ }^{-1}$ (Schleicher et al., 2006). Although the sublimation rate depends on the solar distance, the sublimation fluxes of volatiles for a large part of the $\mathrm{T} 1$ current orbit are higher than the value of $2 \times 10^{5} \mathrm{~kg} \mathrm{yr}^{-1}$ estimated to be due to impacts of asteroids (see De Sanctis et al., 2007). In addition, the mass ejection rate of solid dust due to the sublimation is measured to be $\sim 4 \times 10^{8} \mathrm{~kg} \mathrm{yr}^{-1}$ (Reach et al., 2007), which is also larger than that by the impact. Therefore, the mass loss of the T1 nucleus is not primarily controlled by the collisional processes but by sublimation processes. We suggest that the mass loss by impacts would make only a minor contribution to a decrease in the thickness of a dust mantle. Nevertheless, this does not necessarily mean that the impact is not 
important for the evolution of the T1 nucleus. The collision may play a role to trigger off the formation of active regions on the surface. Britt et al. (2004) and Basilevsky and Keller (2006) proposed that the formation of depression feature, which can be formed by impacts, is an important process on the surface of $\mathrm{T} 1$ to sublime the material below a dust mantle.

We also examine the mass loss rate by using the asteroid population of the BTK model. Similarly, we determine $n(r)$ for the BTK model using $N_{\text {as }}(d)$ as shown in figure 3 in Michel et al. (2009). From the figure, we fit $N_{\text {as }}(d)$ with an assumption of a power-law size distribution as:

$$
N_{\mathrm{as}}(d)=1.6 \times 10^{13}\left(\frac{d}{1 \mathrm{~m}}\right)^{-2.56}, \quad \text { for } d \leq 100 \mathrm{~m}
$$

Considering $d=2 r$, we obtain

$$
n(r)=-\frac{d N_{\mathrm{as}}(d)}{d r}=6.9 \times 10^{12}\left(\frac{r}{1 \mathrm{~m}}\right)^{-3.56} \mathrm{~m}^{-1} .
$$

By using Eq. (7) with the assumptions used above, we obtain

$$
M_{\mathrm{col}} \sim 2.8 \times 10^{4}\left(\frac{r_{\mathrm{max}}}{1 \mathrm{~m}}\right)^{0.44} \mathrm{~kg} \mathrm{yr}^{-1}
$$

The mass loss rate by impacts of asteroidal objects around $\sim 3 \times 10^{4} \mathrm{~kg} \mathrm{yr}^{-1}$ for $r_{\max }=1 \mathrm{~m}$ is much lower than that of water sublimation. Thus, even for the BTK model, the sublimation process dominates over the mass loss by impact.

\section{Collisional History of T1 Nucleus}

\subsection{Number density of craters formed by impacts of} asteroids

We then estimate the collision number into the $\mathrm{T} 1 \mathrm{nu}-$ cleus to infer the collisional history from a comparison with the number of craters observed on $\mathrm{T} 1$ as follows. The total number $N_{\text {col }}$ of impacts by asteroids with radii $\geq r$ into a comet with radius of $R_{\mathrm{T}}$ is given as:

$$
N_{\text {col }}(r)=\int_{r}^{r_{\max }} P_{\mathrm{i}}\left(R_{\mathrm{T}}+r^{\prime}\right)^{2} n\left(r^{\prime}\right) d r^{\prime}
$$

Substituting Eq. (3) into Eq. (9), we obtain

$$
N_{\text {col }}(r) \sim 8.1 \times 10^{-5}\left(\frac{r}{1 \mathrm{~m}}\right)^{-2.88} \mathrm{yr}^{-1},
$$

where we assume $r \ll r_{\max } \ll R_{\mathrm{T}}$.

The rim radius $R_{\mathrm{cr}}$ of a crater formed by an asteroid with radius $r$ can be estimated from Eq. (A.3) as:

$$
R_{\mathrm{cr}}=225\left(\frac{r}{1 \mathrm{~m}}\right)^{0.833} \mathrm{~m}
$$

Using the crater rim diameter $D_{\mathrm{cr}}=2 R_{\mathrm{cr}}$, we can rewrite Eq. (11) as

$$
r=6.5 \times 10^{-4}\left(\frac{D_{\mathrm{cr}}}{1 \mathrm{~m}}\right)^{1.20} \mathrm{~m} .
$$

Substituting Eq. (12) into Eq. (10),

$$
N_{\text {col }}\left(D_{\text {cr }}\right)=1.2 \times 10^{5}\left(\frac{D_{\text {cr }}}{1 \mathrm{~m}}\right)^{-3.46} \mathrm{yr}^{-1} .
$$

From Eq. (13), we estimate that a crater with $D_{\text {cr }}=100 \mathrm{~m}$ is formed at each $\sim 70 \mathrm{yr}$, which is lower than the previous estimate (Yamamoto et al., 2008). This is because these authors used the maximum asteroidal population model by Hawkes and Eaton (2004), which was based on an extrapolation from the population of large observed asteroids $(>\sim 100 \mathrm{~km})$ with a single power-law distribution. However, the population of small asteroids with $r<\sim 100 \mathrm{~m}$ does not follow the extrapolation from the population of large asteroids with $r>\sim 100 \mathrm{~km}$ (see OGR and BTK models).

To compare our results with observational data on T1, we use the $R$-plot defined by equation (10.2.4) in Melosh (1989):

$$
R\left(D_{\mathrm{cr}}\right) \equiv \frac{2^{3 / 4}}{\sqrt{2}-1} D_{\mathrm{cr}}^{2}\left[N_{\mathrm{col}}\left(D_{\mathrm{cr}}\right)-N_{\mathrm{col}}\left(\sqrt{2} D_{\mathrm{cr}}\right)\right] \frac{T}{A},
$$

where $T$ is the exposure age and $A$ is the surface area of the nucleus. The $R$ plot is a conventional method to see the ratio between the actual cumulative number distribution of craters and a cumulative distribution with a slope of -2 , which is thought to be a typical distribution of a saturated crater population on planetary bodies (see e.g., Melosh, 1989). Substituting Eq. (13) into Eq. (14), we obtain

$$
R\left(D_{\mathrm{cr}}\right)=3.1 \times 10^{-3}\left(\frac{D_{\mathrm{cr}}}{1 \mathrm{~m}}\right)^{-1.46}\left(\frac{T}{1 \mathrm{yr}}\right) .
$$

In Fig. 1, we plot $R\left(D_{\mathrm{cr}}\right)$ against $D_{\mathrm{cr}}$ for various $T$. Also plotted are the observational data of likely impact crater remnants on T1 by Thomas et al. (2007). From this figure, we estimate $T$, although the observational data intersects several lines with various $T$. We first consider that $T$ must be longer than 300 years. While the $R$ value for the largest craters $\left(D_{\mathrm{cr}}=400 \mathrm{~m}\right)$ shows an older age of $T \sim 10^{5}$ years, the data for the largest few craters may have a stochastic problem. Namely, there are too few the largest craters to give us an exact age. If we exclude the data with $D_{\text {cr }}=400 \mathrm{~m}$, the $R$ values for craters with $D_{\text {cr }}>\sim 150 \mathrm{~m}$ seem to follow the line with $T=10^{4}$ years. In contrary, the $R$ value for smaller craters with $D_{\text {cr }}<100 \mathrm{~m}$ shows $T<10^{4}$ years, but this may be due to the sublimation process, as discussed later. Therefore, it is likely that the time necessary to accumulate the crater population on the T1 nucleus exceeds $\sim 10^{4}$ years (up to $10^{5}$ years).

Numerical calculations suggest that before T1 had close approaches to Jupiter $\sim 300-400$ years ago, the perihelion of T1 may have been further than the asteroid belt region (e.g., Yeoman et al., 2005). However, this does not necessarily mean that $\mathrm{T} 1$ has never crossed the asteroid belt region prior to $\sim 400$ years ago. Levison and Duncan (1997) reported that typical Jupiter-Family Comets (JFCs) change their perihelion and aphelion distances frequently after becoming JFCs. Thus, it is likely that T1 may have crossed the asteroid belt region many times in the past, forming craters corresponding to the $R$-plot for at least $10^{4}$ years. Note that the actual duration time may be longer (or shorter) than $10^{4}$ years, because we used the values of $P_{\mathrm{i}}$ and $v_{\mathrm{i}}$ for the present orbit of T1 (see Table 1), which might have been smaller (or larger) than the present values. 


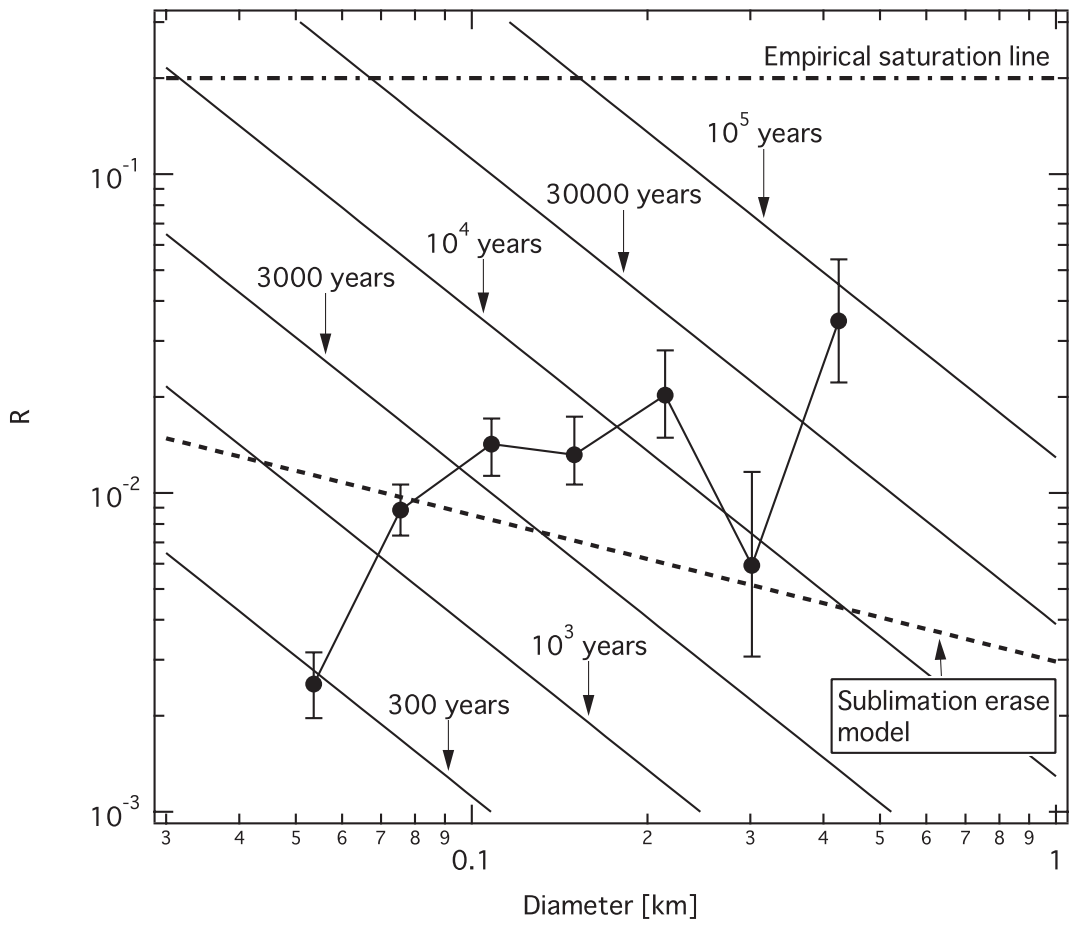

Fig. 1. $R$-plot against crater diameter for various exposure age $T$. Solid circles indicate the data observed on T1 (Thomas et al., 2007). Dash-dotted line indicates the empirical saturation line $(R \sim 0.2)$. Dashed-line indicates the $R$ value for our sublimation erase model in Eq. $(22)$.

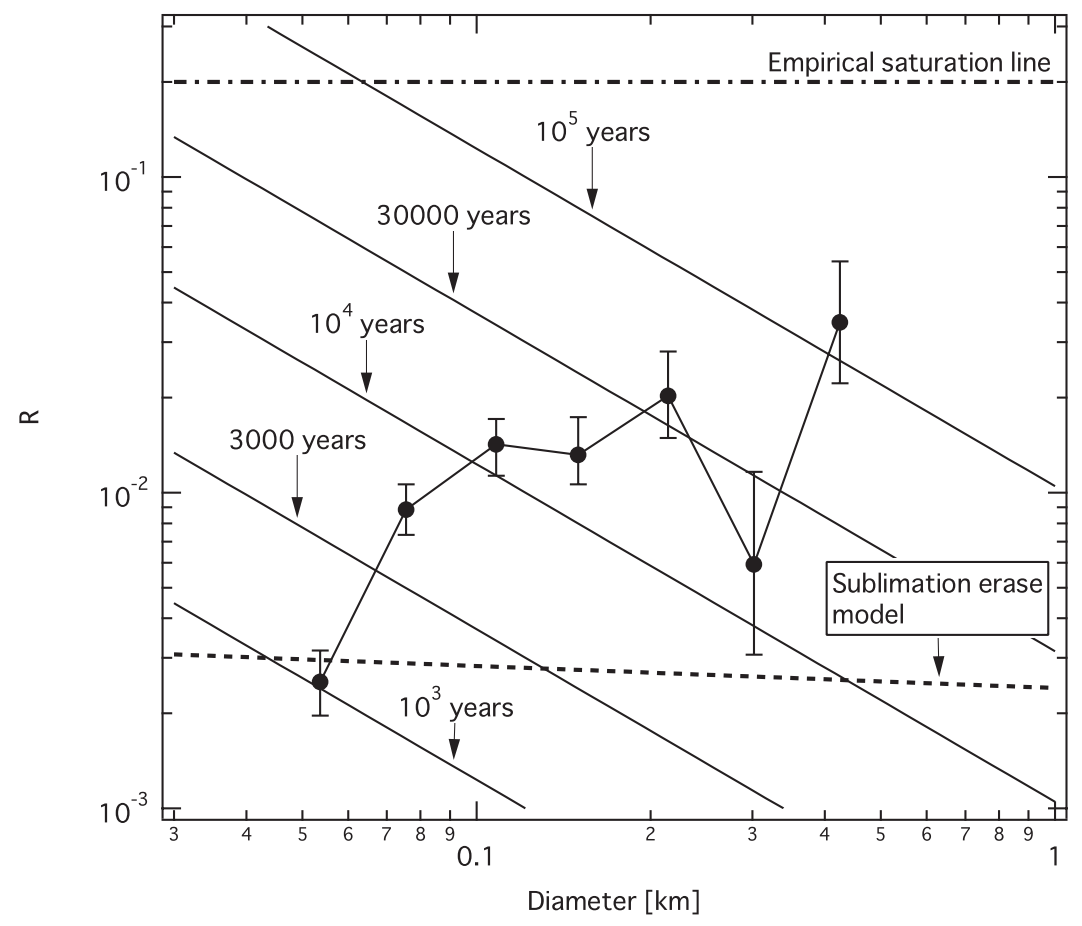

Fig. 2. The same as Fig. 1, but for the BTK model. Dash-dotted line indicates the empirical saturation line $(R \sim 0.2)$. Dashed line indicates the $R$ value for sublimation erase model as: $R\left(D_{\mathrm{cr}}\right)=3.9 \times 10^{-3}\left(\frac{D_{\mathrm{cr}}}{1 \mathrm{~m}}\right)^{-0.07}$, which is derived from Eqs. (16) and (21).

We also examine the case for the BTK model. Using Eq. (7), we also derived

$$
R\left(D_{\mathrm{cr}}\right)=1.7 \times 10^{-4}\left(\frac{D_{\mathrm{cr}}}{1 \mathrm{~m}}\right)^{-1.07}\left(\frac{T}{1 \mathrm{yr}}\right) .
$$

In Fig. 2, we plot $R\left(D_{\text {cr }}\right)$ against $D_{\text {cr }}$ for various $T$. Also plotted are the observational data on T1 regarded as impact crater remnants by Thomas et al. (2007). We can see that the $R$ values for craters with $D_{\text {cr }} \sim 100-300 \mathrm{~m}$ seem to follow the line with $T=10^{4}$ to $\sim 3 \times 10^{4}$ years, and the $R$ value for the largest craters $\left(D_{\mathrm{cr}}=400 \mathrm{~m}\right)$ shows an older age of $T \sim 10^{5}$ years. Thus, even for the BTK model, the time necessary to accumulate the crater population on the T1 nucleus exceeds $\sim 10^{4}$ years (up to $10^{5}$ years), which is 
almost the same result as that for the OGR model.

3.2 Effects of sublimation process on crater population

As we see in Section 2, the mass loss by the sublimation process is more significant than that by impacts of asteroids. In this case, we may consider the possibility that the sublimation process erases or changes the crater population. Our question is: how would the sublimation process change the discussion in Section 3.1? We thus investigate the effect of sublimation on the crater population of the T1 surface hereafter.

We first estimate the thickness $H_{\mathrm{s}}$ of the T1 surface lost by sublimation as follows. The sublimation rate of $6 \times 10^{27}$ water molecules $\mathrm{s}^{-1}$ is much higher than that expected from the water spot regions on the T1 nucleus (a few \% in area) (see e.g., Schleicher et al., 2006; De Sanctis et al., 2007). We thus assume that dust particles are ejected not only from the active regions but also from the dust mantle by the sublimation of water vapor that passes through the dust mantle during the sublimation process. Assuming homogeneous sublimation from the whole surface of the T1 nucleus, we estimate the total mass lost by sublimation during $T_{\mathrm{s}}$ as

$$
4 \pi R_{\mathrm{T}}^{2} H_{\mathrm{s}} \rho_{\mathrm{t}}=\dot{M}_{\mathrm{s}} T_{\mathrm{s}},
$$

where $\dot{M}_{\mathrm{s}}$ is the mass-loss rate of surface materials due to sublimation. From Eq. (17), the thickness $H_{\mathrm{s}}$ can be written as

$$
\begin{aligned}
H_{\mathrm{s}} & =\frac{\dot{M}_{\mathrm{s}} T_{\mathrm{s}}}{4 \pi \rho_{\mathrm{t}} R_{\mathrm{T}}^{2}} \\
& =8.7 \times 10^{-3}\left(\frac{\dot{M}_{\mathrm{s}}}{14 \mathrm{~kg} \mathrm{~s}^{-1}}\right)\left(\frac{T_{\mathrm{s}}}{1 \mathrm{yr}}\right) \mathrm{m} .
\end{aligned}
$$

The mass loss rate of large dust particles due to sublimation is reported to be $\dot{M}_{\mathrm{s}} \sim 14 \mathrm{~kg} \mathrm{~s}^{-1}$ (Reach et al., 2007). In this case, we estimate $H_{\mathrm{s}} \sim 2.6 \mathrm{~m}$ for $T=300$ years. If we use $\dot{M}_{\mathrm{s}} \sim 180 \mathrm{~kg} \mathrm{~s}^{-1}$ for the water sublimation rate (e.g., Schleicher et al., 2006), we estimate $H_{\mathrm{s}} \sim 34 \mathrm{~m}$ for $T=300$ years. Moreover, Lisse et al. (2005) reported a twofold decrease in the water sublimation rate from 1983 to 1994 , suggesting that the rate of water sublimation in the past was higher than the present rate. Therefore, the $\mathrm{T} 1$ primordial top layer of at least several meters could have been lost owing to the sublimation process. Namely, any primordial materials on the surface layers of T1 (e.g., residue created in trans-Neptunian region) cannot remain on the surface.

We next estimate to what degree craters are erased by the sublimation process. Consider that a crater with depth $h_{\text {cr }}$ and diameter $D_{\text {cr }}$ is completely erased when $H_{\mathrm{s}}=h_{\mathrm{cr}}$. Namely,

$$
H_{\mathrm{s}}=h_{\mathrm{cr}}=f D_{\mathrm{cr}},
$$

where $f$ is a depth-diameter ratio of a crater. Hereafter, we call this sublimation-driven process of erasing craters "sublimation erase model”. Substituting Eq. (19) into Eq. (20), we obtain the time $T_{\text {er }}$ needed to erase a crater with diameter $D_{\text {cr }}$ as

$$
T_{\mathrm{er}}=23\left(\frac{D_{\mathrm{cr}}}{1 \mathrm{~m}}\right) \quad \mathrm{yr},
$$

where we assume $\dot{M}_{\mathrm{s}}=14 \mathrm{~kg} \mathrm{~s}^{-1}$ (Reach et al., 2007) and adopt $f=0.2$, which was measured for the crater population on 81P/Wild 2 nucleus (Basilevsky and Keller, 2006). Thus, when $T_{\mathrm{s}}>T_{\mathrm{er}}$, the number of craters with diameter of $D_{\text {cr }}$ remaining on the T1 surface is suppressed to $N_{\text {col }}\left(D_{\text {cr }}\right) \times T_{\text {er. }}$. Substituting Eq. (21) into Eq. (15), we obtain the $R-D_{\text {cr }}$ relation for the sublimation erase model as

$$
R\left(D_{\mathrm{cr}}\right)=7.1 \times 10^{-2}\left(\frac{D_{\mathrm{cr}}}{1 \mathrm{~m}}\right)^{-0.46} \text { for } D_{\mathrm{cr}} \leq \frac{1}{23}\left(\frac{T_{\mathrm{s}}}{1 \mathrm{yr}}\right) \mathrm{m} .
$$

Equation (22) is also plotted in Fig. 1 (dashed-line). We see that the observational data with $D_{\mathrm{cr}}>\sim 100 \mathrm{~m}$ are located above the line of Eq. (22). This indicates that the total duration of sublimation is $T_{\mathrm{s}}<\sim 2 \times 10^{3}$ years or that $\dot{M}_{\mathrm{s}}$ in the past was lower than the value used here. In any case, this may suggest that the duration of the present significant sublimation is less than $\sim 2 \times 10^{3}$ years.

We see that the observational data with $D_{\text {cr }} \sim 50 \mathrm{~m}$ is less than that by the sublimation erase model in Fig. 1 . This may be explained as follows. First, water sublimation under the dust mantle may also contribute to erasing such small craters. The dust mantle layer would subside when the water ices below the dust mantle are lost by the sublimation process. During this subsidence, some of the small craters might have collapsed. Second, involved with the escape of water vapor through the dust mantle, the shape of small craters may be deformed too much to be identified as crater features. Third, large-sized dust fallen back to the surface may cover small craters. Fourth, the redistribution of regolith by seismic-shaking induced by large impacts proposed by Richardson et al. (2005) may erase small craters. The decrease in the $R$ value for small craters is a typical feature for the small crater population with $D<\sim 100 \mathrm{~m}$ of near-Earth asteroids, such as Eros or Itokawa, which can be explained by a smoothing effect from the redistribution of regolith by the seismic-shaking (e.g., Richardson et al., 2005; Michel et al., 2009). However, the report by Thomas et al. (2007) that T1 has higher surface slopes than other small bodies suggests a lack of the smoothing effect from the redistribution of regolith. In any case, although it is difficult to uniquely attribute the erasure of craters to one of the above-mentioned processes, some erase processes may be needed to explain the small crater population on the $\mathrm{T} 1$ nucleus.

\subsection{Possible scenario for the evolution of T1}

From the above discussion, we expect the following scenario for T1. T1 might have become JFC $\sim 10^{4}-10^{5}$ years ago. T1 subsequently changed the perihelion distance as well as the aphelion distance many times, which was predicted by numerical calculations for JFCs (Levison and Duncan, 1994, 1997). During this period, T1 crossed the asteroid-belt frequently to accumulate the observed crater population on the surface. Recent close approaches to Jupiter about $\sim 400$ years ago changed the T1 perihelion to be $\sim 1.5 \mathrm{AU}$ (Yeomans et al., 2005). During this period, the smaller crater population decreased owing to significant sublimation. We cannot rule out the possibility that T1 suffered from significant sublimation before 400 years ago, but the total duration time of such significant sublimation is most likely less than $\sim 2 \times 10^{3}$ years.

This scenario is based on the assumption that crater size 
does not change during the sublimation process. On the other hand, crater sizes may increase with time, because the sublimation process may erode the inside wall of craters, as suggested by Britt et al. (2004). From Eq. (19) with the water sublimation of $\dot{M}_{\mathrm{s}}=180 \mathrm{~kg} \mathrm{~s}^{-1}$, the retreat rate by sublimation may be $\sim 0.11 \mathrm{~m} \mathrm{yr}^{-1}$. Thus, we estimate that the increase in $D_{\text {cr }}$ by sublimation is $\sim 88 \mathrm{~m}$ for 400 years. In this case, the original crater sizes of $D_{\mathrm{cr}}=200-300 \mathrm{~m}$ (with $T \sim 10^{4} \mathrm{yr}$ ) might have been $D_{\mathrm{cr}} \sim 100-200 \mathrm{~m}$, which corresponds to $T \sim 2000-5000$ years estimated from Eq. (15), i.e., $T \propto D_{\mathrm{cr}}^{1.46}$. Thus, the age that T1 crossed the asteroid belt region may be as short as several thousands of years. Nevertheless, there is currently no clear correlation between the active regions and the impact craters on $\mathrm{T} 1$ (see Thomas et al., 2007). If the sublimation process eroded the inside wall of craters, we would observe many active regions inside the craters. This is, however, not the case. We expect that craters formed by asteroid impacts can be eroded by the sublimation process and become bigger, but the sublimation process would be quenched immediately owing to the formation of a dust mantle on the crater wall. The increase in the crater size by sublimation may not change significantly the above scenario for $\mathrm{T} 1$.

We also assume that there are no ancient craters formed by impacts with the other trans-Neptunian objects before T1 entered the inner Solar System. The reasoning for this is as follows. If the observed craters were ancient craters formed in the trans-Neptunian region, a large number of impacts with trans-Neptunian objects may be expected (e.g., Stern, 1996), and the $R$ value for T1 would be around the empirical saturation line with $R \sim 0.2$. 81P/Wild 2 is an example showing that the most of crater population follow the empirical saturation line (Basilevsky and Keller, 2006). It has been suggested that the surface of $81 \mathrm{P} /$ Wild 2 nuclei remains very old ancient terrain, presumably dating back to the comet's residence in the trans-Neptunian region (e.g., Brownlee et al., 2004). Furthermore, while 81P/Wild 2 has many large craters with $D_{\mathrm{cr}}>400 \mathrm{~m}$, there are no large craters with $D_{\text {cr }}>400 \mathrm{~m}$, suggesting that the T1 surface is not old enough to accumulate such large craters. We conclude that resurface events over the T1 surface in the past might have erased most of ancient craters on $\mathrm{T} 1$.

\section{Summary}

We have studied the mass-loss rate of surface materials by impacts of asteroidal objects. The mass-loss rate by impacts of asteroidal objects is around $\sim 2 \times 10^{5} \mathrm{~kg} \mathrm{yr}^{-1}$, which is smaller than that of the water sublimation of $\sim 6 \times 10^{9} \mathrm{~kg} \mathrm{yr}^{-1}$. This result suggests that the mass loss of a comet nucleus is mainly due to sublimation processes, and such a significant sublimation process has lost most, if not all, of the primordial materials on the surface layers of T1. However, this does not necessarily mean that the impact is not important for the evolution of the T1 nucleus because the collision process may play a role in triggering the formation of active regions on the surface. We have then estimated the number density of craters by asteroid impacts and compared this with the observational data. We found that the time necessary to accumulate the crater population on $\mathrm{T} 1$ is longer than 300 years, probably $\sim$ a few $10^{4}$ years, suggesting that $\mathrm{T} 1$ crossed the asteroid belt region during at least $\sim 10^{4}$ years in the past. If the crater size increases with time owing to sublimation, this age may be as short as several thousands of years. The present sublimation rate for $\mathrm{T} 1$ is very significant because of the recent orbit close to the Sun. A large number of small craters would be erased by such significant sublimation processes. Comparing the number density of craters with the sublimation erase model, we estimate that the timing of T1's entry into the recent orbit may be later than $\sim 2 \times 10^{3}$ years ago.

Acknowledgments. The authors thank A. M. Nakamura for helpful comments, and D. Durda and D. O'Brien for giving valuable comments and suggestions as reviewers. This study was supported in part by T. Yoda, and Grant-in-Aid for Young Scientists (B) (20740249; 20740247).

\section{Appendix A. Scaling Formulae for the Crater Rim Radius and the Total Mass of Impact Ejecta Escaping from a Target Body}

In this appendix, we derive the formulae for a crater rim radius $R_{\mathrm{cr}}$, which is defined by the distance from the center to the rim of the crater, and for the total mass of ejecta $m_{\mathrm{e}}$ escaping from a target body, provided that an object of radius $r$ and density $\rho_{\mathrm{i}}$ (then the mass $m_{\mathrm{i}}=4 \pi \rho_{\mathrm{i}} r^{3} / 3$ ) impacts with velocity $v_{\mathrm{i}}$ onto the comet surface of density $\rho_{\mathrm{t}}$ and gravity $g$. We assume that the cratering process is in the gravity regime, namely independent of the material strength (see Holsapple, 1993).

The apparent crater radius $R_{\text {at }}$, which is measured along a pre-impact surface level, is expressed in the gravity regime (e.g., Housen et al., 1983) as:

$$
R_{\mathrm{at}}=K_{1}\left(3.22 \frac{g r}{v_{\mathrm{i}}^{2}}\right)^{-\epsilon_{\mathrm{x}}}\left(\frac{m_{\mathrm{i}}}{\rho_{\mathrm{t}}}\right)^{\frac{1}{3}},
$$

where $K_{1}$ and $\epsilon_{\mathrm{x}}$ are constants. When we use the values of $\epsilon_{\mathrm{x}}=0.167$ and $K_{1}=0.85$ for craters formed in Ottawa sand (Schmidt, 1980), we obtain

$$
R_{\mathrm{at}}=1.13\left(\frac{g r}{v_{\mathrm{i}}^{2}}\right)^{-0.167}\left(\frac{\rho_{\mathrm{i}}}{\rho_{\mathrm{t}}}\right)^{\frac{1}{3}} r .
$$

Assuming the rim diameter of a crater is 1.3 times larger than the apparent diameter (Housen et al., 1983; Holsapple, 1993), the rim radius is given by

$$
R_{\mathrm{cr}}=1.47\left(\frac{g r}{v_{\mathrm{i}}^{2}}\right)^{-0.167}\left(\frac{\rho_{\mathrm{i}}}{\rho_{\mathrm{t}}}\right)^{\frac{1}{3}} r .
$$

Housen et al. (1983) formulated the volume $V_{\mathrm{t}}\left(>v_{\mathrm{e}}\right)$ of impact ejecta with velocity higher than $v_{\mathrm{e}}$ in the gravity regime as:

$$
\frac{V_{\mathrm{t}}\left(>v_{\mathrm{e}}\right)}{R_{\mathrm{at}}^{3}}=K_{2}\left(\frac{v_{\mathrm{e}}}{\sqrt{g R_{\mathrm{at}}}}\right)^{-\epsilon_{\mathrm{v}}}
$$

where $K_{2}$ and $\epsilon_{\mathrm{v}}$ are constants. According to Housen et al. (1983), $\epsilon_{\mathrm{v}}$ and $\epsilon_{\mathrm{x}}$ are related each other by the formula $\epsilon_{\mathrm{v}}=6 \epsilon_{\mathrm{x}} /\left(1-\epsilon_{\mathrm{x}}\right)$. Using Eq. (A.4), the total mass of the escaping ejecta $m_{\mathrm{e}}$ is expressed as

$$
\begin{aligned}
m_{\mathrm{e}} & =V_{\mathrm{t}}\left(>v_{\mathrm{e}}\right) \rho_{\mathrm{t}} \\
& =K_{2}\left(\frac{v_{\mathrm{e}}}{\sqrt{g R_{\mathrm{at}}}}\right)^{-\epsilon_{\mathrm{v}}} R_{\mathrm{at}}^{3} \rho_{\mathrm{t}} .
\end{aligned}
$$


Substituting Eq. (A.2) into Eq. (A.6), we obtain

$$
m_{\mathrm{e}}=0.49\left(\frac{v_{\mathrm{i}}}{v_{\mathrm{e}}}\right)^{1.20}\left(\frac{\rho_{\mathrm{i}}}{\rho_{\mathrm{t}}}\right)^{1.20} \rho_{\mathrm{t}} r^{3},
$$

where we use the values of $K_{2}=0.32$ and $\epsilon_{\mathrm{v}}=$ $6 \epsilon_{\mathrm{x}} /\left(1-\epsilon_{\mathrm{x}}\right)=1.20$ for cratering processes in the gravity regime (Housen et al., 1983).

\section{References}

A’Hearn, M. F., M. J. S. Belton, W. A. Delamere, J. Kissel, K. P. Klaasen, L. A. McFadden, K. J. Meech, H. J. Melosh, P. H. Schultz, J. M. Sunshine, P. C. Thomas, J. Veverka, D. K. Yeomans, M. W. Baca, I. Busko, C. J. Crockett, S. M. Collins, M. Desnoyer, C. A. Eberhardy, C. M. Ernst, T. L. Farnham, L. Feaga, O. Groussin, D. Hampton, S. I. Ipatov, J.-Y. Li, D. Lindler, C. M. Lisse, N. Mastrodemos, W. M. Owen Jr., J. E. Richardson, D. D. Wellnitz, and R. L. White, Deep Impact: Excavating comet Tempel 1, Science, 310, 258-264, 2005.

Bar-Nun, A., E. Heifetz, and D. Prialnik, Thermal evolution of Comet $\mathrm{P} /$ Tempel 1 -Representing the group of targets for the CRAF and CNSR missions, Icarus, 79, 116-124, 1989.

Basilevsky, A. T. and H. U. Keller, Comet nuclei: Morphology and implied processes of surface modification, Planet. Space Sci., 54, 808-829, 2006.

Bottke, W. F., D. D. Durda, D. Nesvorný, R. Jedicke, A. Morbidelli, D. Vokrouhlický, and H. Levison, The fossilized size distribution of the main asteroid belt, Icarus, 175, 111-140, 2005.

Britt, D. T., D. C. Boice, B. J. Buratti, H. Campins, R. M. Nelson, J. Oberst, B. R. Sandel, S. A. Stern, L. A. Soderblom, and N. Thomas, The morphology and surface processes of Comet 19/P Borrelly, Icarus, 167, 45-53, 2004.

Brownlee, D. E., F. Horz, R. L. Newburn, M. Zolensky, T. C. Duxbury, S. Sandford, Z. Sekanina, P. Tsou, M. S. Hanner, B. C. Clark, S. F. Green, and J. Kissel, Surface of young Jupiter family comet 81P/Wild 2: view from the stardust spacecraft, Science, 304, 1764-1769, 2004.

Davidsson, B. J. R., P. J. Gutiérrez, and H. Rickman, Nucleus properties of Comet 9P/Tempel 1 estimated from non-gravitational force modeling, Icarus, 187, 306-320, 2007.

De Sanctis, M. C., M. T. Capria, A. Coradini, and E. Ammannito, Thermal evolution models of the 9P/Tempel 1 comet nucleus for interpreting the Deep Impact results, AJ, 133, 1836-1846, 2007.

Fernández, J. A., The role of collisions with interplanetary particles in the physical evolution of comets, Moon Planets, 25, 507-519, 1981.

Gil-Hutton, R., Collisional rates and impact velocities of short-period comets with interplanetary particles, Planet. Space Sci., 48, 545-551, 2000.

Gronkowski, P., Cometary outbursts-the post-Deep Impact outlook on collisions as possible causes, Mon. Not. R. Astron. Soc., 379(3), 1049_ 1052, 2007.

Hawkes, R. L. and R. A. Eaton, Mass flux of asteroidal origin meteoroids on periodic comet nuclei, Earth Moon Planets, 95, 187-195, 2004.

Holsapple, K. A., The scaling of impact processes in planetary sciences, Ann. Rev. Earth Planet. Sci., 21, 333-373, 1993.

Housen, K. R., R. M. Schmidt, and K. A. Holsapple, Crater ejecta scaling laws: Fundamental forms based on dimensional analysis, J. Geophys. Res., 88, 2485-2499, 1983.

Jewitt, D., Physical properties of cometary nuclei, in Observations and Physical Properties of Small Solar System Bodies, edited by A. Brahic, J.-C. Gerard, and J. Surdej. (Liège Universite de Liège), 85-112, 1992.
Lisse, C. M., M. F. A'Hearn, T. L. Farnham, O. Groussin, K. J. Meech, U. Fink, and D. G. Schleicher, The coma of comet 9P/Tempel 1, Space Sci. Rev., 117, 161-192, 2005.

Levison, H. F. and M. J. Duncan, The long-term dynamical behavior of short-period comets, Icarus, 108, 18-36, 1994.

Levison, H. F. and M. J. Duncan, From the Kuiper belt to Jupiter-family comets: The spatial distribution of ecliptic comets, Icarus, 127, 13-32, 1997.

Meech, K. J., Cometary origin and evolution, in A New Era in Bioastronomy, edited by G. Lemarchand and K. Meech, 213, 207-215, 2000.

Melosh, H. J., Impact Cratering, Oxford Univ. Press, New York, 1989.

Michel, P., D. P. O'Brien, S. Abe, and N. Hirata, Itokawa's cratering record as observed by Hayabusa: Implications for its age and collisional history, Icarus, 200, 503-513, 2009.

Mumma, M. J., M. A. DiSanti, K. Magee-Sauer, B. P. Bonev, G. L. Villanueva, H. Kawakita, N. Dello Russo, E. L. Gibb, G. A. Blake, J. E. Lyke, R. D. Campbell, J. Aycock, A. Conrad, and G. M. Hill, Parent volatiles in comet 9P/Tempel 1: Before and after impact, Science, 310, 270-274, 2005.

O'Brien, D. P. and R. Greenberg, The collisional and dynamical evolution of the main-belt and NEA size distributions, Icarus, 178, 179-212, 2005.

O'Brien, D. P., R. Greenberg, and J. E. Richardson, Craters on asteroids: Reconciling diverse impact records with a common impacting population, Icarus, 183, 79-92, 2006.

Prialnik, D., J. Benkhoff, and M. Podolak, Modeling the structure and activity of comet nuclei, in Comets II, edited by M. C. Festou, H. U. Keller, and H. A. Weaver, 359-387 pp., Univ. of Arizona Press, 2004.

Prialnik, D., G. Sarid, E. D. Rosenberg, and R. Merk, Thermal and chemical evolution of comet nuclei and Kuiper belt objects, Space Sci. Rev., doi:10.1007/s11214-007-9301-4, 2008.

Reach, W. T., M. S. Kelley, and M. V. Sykes, A survey of debris trails from short-period comets, Icarus, 191, 298-322, 2007.

Richardson Jr., J. E., H. J. Melosh, R. J. Greenberg, and D. P. O'Brien, The global effects of impact-induced seismic activity on fractured asteroid surface morphology, Icarus, 179, 325-349, 2005.

Schleicher, D. G., K. L. Barnes, and N. F. Baugh, Photometry and imaging results for comet 9P/Tempel 1 and Deep Impact: Gas production rates, postimpact light curves, and ejecta plume morphology, Astron. J., 131, 1130-1137, 2006.

Schmidt, R. M., Meteor Crater: Energy of formation-implications of centrifuge scaling, Proc. Lunar Planet. Sci. Conf. 11th, 2099-2128, 1980.

Stern, S. A., Signatures of collisions in the Kuiper Disk, Astron. Astrophys., 310, 999-1010, 1996.

Sykes, M. V. and R. G. Walker, Cometary dust trails. I - Survey, Icarus, 95, 180-210, 1992.

Thomas, P. C., J. Veverka, M. J. S. Belton, A. Hidy, M. F. A'Hearn, T. L. Farnham, O. Groussin, J.-Y. Li, L. A. McFadden, J. Sunshine, D. Wellnitz, C. Lisse, P. Schultz, K. J. Meech, and W. A. Delamere, The shape, topography, and geology of Tempel 1 from Deep Impact observations, Icarus, 187, 4-15, 2007.

Yamamoto, S., H. Kimura, E. Zubko, H. Kobayashi, K. Wada, M. Ishiguro, and T. Matsui, Comet 9P/Tempel 1: Interpretation with the Deep Impact results, Astrophys. J., 673, L199-L202, 2008.

Yeomans, D. K., J. D. Giorgini, and S. R. Chesley, The history and dynamics of comet 9P/Tempel 1, Space Sci. Rev., 117, 123-135, 2005.

S. Yamamoto (e-mail: yamachan@gfd-dennou.org), K. Wada, H. Kobayashi, H. Kimura, M. Ishiguro, and T. Matsui 